\title{
Ventilator-associated pneumonia (VAP) caused by Acinetobacter baumannii in view of the microbial properties of the ESKAPE group in neighbouring countries - Poland and Ukraine
}

\author{
Aneta Grochowalska', Maria Kozioł-Montewka², Oleksandr Oliynyk ${ }^{3}$, Natalia Krasij ${ }^{4}$ \\ ${ }^{1}$ Laboratory of Microbiology, Masovian Specialistic Hospital in Radom, Poland \\ 2 Pope John Paul II State School of Higher Education in Biała Podlaska, Poland \\ ${ }^{3}$ I. Horbachevsky Ternopil State Medical University, Ukraine \\ ${ }^{4}$ Ternopil Medical University Hospital, Ternopil, Ukraine
}

Grochowalska A, Kozioł-Montewka M, Oliynyk O, Krasij N. Ventilator-associated pneumonia (VAP) caused by Acinetobacter baumannii in view of the microbial properties of the ESKAPE group in neighbouring countries - Poland and Ukraine. J Pre-Clin Clin Res. 2017 ; $11(2)$ : $111-115$. doi: $10.26444 / j p c c r / 76024$

\begin{abstract}
The increase in the clinical threat of ESKAPE pathogens is associated with high virulence and the presence of numerous mechanisms of antimicrobial resistance. The above characteristics of pathogens affect therapeutic failures as well as cause prolongation and increase of the cost of its treatment.

Objective. The aim of this study was to evaluate and analyze Acinetobacter baumannii strains in relation to the remaining bacteria from the ESKAPE group that induced pneumonia in mechanically ventilated patients in two neighboring countries. Materials and method. The study was retrospective. 335 clinical materials were analyzed, mainly bronchoalveolar lavage gained for microbiological screen. The analyzed patients were in intensive care units (ICU) in selected hospitals in 2013 2015, in Poland (154 patients) and in Ukraine (181 patients).

Results. In the analysis of etiological agents of VAP caused by ESKAPE strains, Gram negative bacteria were predominant in both countries although particular species occurred at different frequencies in Poland and Ukraine. Multi-resistant strains of Acinetobacter baumannii were more frequently cultured from patients in Poland (26.9\%) compared to those from Ukraine (14.6\%).
\end{abstract}

\section{Key words}

Acinetobacter baumannii, ESKAPE, VAP, resistance to antibiotics

\section{INTRODUCTION}

The pathogens that cause severe hospital infections in all countries of the world, contributing to the highest mortality rates, are those with high resistance to antibiotics. These organisms, by their rapid expansion, have become a global threat to public health. For the purposes of epidemiological studies, different names for resistant strains have been defined:

- MDR - multidrug resistance

- XDR - extensively drug resistance

- PDR - pandrug resistance

The groups of pathogens include Enterococcus faecium, Staphylococcus aureus, Klebsiella pneumoniae, Acinetobacter baumannii, Pseudomonas aeruginosa and Enterobacter spp. These microorganisms were identified by the IDSA (Infectious Diseases Society of America) with the acronym ESKAPE $[1,2,3]$. Acquired resistance genes of these bacteria are manifested by the lack of efficacy of vancomycin against Enterococcus-resistant enterococci (VRE), resistance to $S$. aureus (MRSA) and antibiotic resistance to $\beta$-lactam antibiotics, mainly against Gram-negative carbapenems

Address for correspondence: Aneta Grochowalska, Laboratory of Microbiology, Masovian Specialistic Hospital in Radom, Poland, Aleksandrowicza 5, 26-617 Radom, Poland

E-mail: aneta.grochowalska@op.pl

Received: 10 May2017; accepted: 23 July 2017 (e.g. CRE - carbapenem-resistant Enterobacteriaceae) $[4,5]$. The effect of carbapenemases production among clinically significant Gram-negative bacteria is a serious medical problem. So far, Acinetobacter baumannii has been identified as the final recipient of carbapenemase genes (e.g. imipenemase, Veron metallo- $\beta$-lactamase, Guiana extended spectrum and carbapenemase Klebsiella pneumoniae) from Enterobacteriaceae and Pseudomonas aeruginosa. Recent discoveries concerning the proliferation of blaNDM carbapenemase genes have shown that A. baumannii probably acts as a source of genes resistant to antibiotics. The genetic analysis of blaNDM-1 showed that the genetic structure (Tn125) responsible for its propagation is most likely derived from Acinetobacter. Furthermore, blaNDM-1 studies showed that it can be constructed in Acinetobacter by a recombinant event with another resistance gene found in A. baumannii (aphA6). This way of looking at it highlights the new and unexpected role of $A$. baumannii [6].

In the last two decades, Acinetobacter infection has increased. One reason for this is a high level of natural and acquired resistance to many antimicrobials and disinfectants. The widespread occurrence of $A$. baumannii in the hospital environment, mostly in humid areas or colonizing patients' mucous membranes and elements of therapeutic devices, such as intubation tubes or components of artificial ventilation devices, cause frequent infections, especially in intensive care units. The low nutrient requirements of Acinetobacter enable 
the bacteria to remain in the dry environment for weeks or months. The described microbial properties are often associated with surface contamination in hospitals, causing epidemical outbreaks $[7,8]$. Neither adverse environmental conditions nor the problem of biofilm formation should be overlooked, as the microorganisms gain an exceptionally high level of antimicrobial resistance $[4,9]$. The effects of various factors, such as inappropriate and excessive antibiotic therapy, invasive medical diagnostic and therapeutic procedures, inadequate hygiene, and international and intercontinental travel, would most likely lead to the rapid growth and spread of these bacteria in hospitals $[10,11]$.

Developed internal epidemiological procedures allow evaluation of the possibility of the spread of these bacteria within respective hospitals. National molecular studies allow determination of the spread of resistant strains in the country. However, there is little research analyzing the relationship between antibiotic regimens in different countries, the overall clinical status of patients, and the prevalence of antibioticresistant strains.

\section{OBJECTIVES}

The aim of the study was to evaluate and analyze the incidence of Acinetobacter baumannii strains with respect to the remaining ESKAPE bacteria in ventilator-associated pneumonia (VAP) patients in a selected hospital in Poland (Radom, Masovian Specialist Hospital) and a comparable university hospital in Ternopil, Ukraine.

Patients were also evaluated in terms of clinical data predisposing the development of severe pneumonia in both examination groups.

The recommended empirical antibiotic regimens and the practical use of selected antibiotics in both countries were compared with established Acinetobacter resistance.

\section{MATERIALS AND METHOD}

The study was retrospective. 335 clinical samples were analyzed: bronchoalveolar lavage (BAL) collected for microbiological screening from patients undergoing artificial ventilation who developed pneumonia. The analyzed patients were in intensive care units (ICU) in 2013-2015: 154 patients in Poland (PL) (Masovian Specialist Hospital in Radom) and in Ukraine 181 patients (UA) (Hospital at Ternopil State Medical University).

Methodology of microbiological diagnostics. In Poland, the same as in Ukraine, microbiological diagnostics of lower respiratory tract materials was conducted as a quantitative culture of secretion. One of the criteria for laboratory diagnosis of pneumonia was the number and morphology of bacteria in the direct preparation and the culture of bronchial secretions in the quantitative culture $>10^{4} \mathrm{CFU} / \mathrm{ml}$ bacterial strains obtained by BAL method. Subsequently, the material was seeded according to general principles of microbiological diagnostics. For the cultivation, ready bacteriological mediums of bioMerieux agars were used: Columbia Agar with 5\% Sheep Blood, McConkey Agar, Chapman Agar, Haemophilus Chocolate Agar. Incubation was carried out under oxygen conditions and $\mathrm{CO}_{2}$-enriched atmosphere at $36^{\circ} \mathrm{C} \pm 1{ }^{\circ} \mathrm{C}$; bacterial growth was then observed after $18-24$ hours. Subsequently, after determining the morphology of the colonies and after microscopic observations, the species was identified by means of commercial kits: in Poland ID cards GN of Vitek 2 Compact apparatuses; in Ukraine, ENTEROtest 24 STAFITEST 16 test systems (Pliva-Lachema, Czech Republic) and VITEK 2 Compact automatic analyzer (BioMerieux, France).

In the two countries, there was also pre-identification of non-fermenting Gram-negative bacteria, including Pseudomonas aeruginosa and Acinetobacter spp. Analyses were carried out on Hugh-Leifson's medium, where glucose oxidation could be observed. A test to detect the cytochrome oxidase enzyme was then performed. $P$. aeruginosa bacteria produced oxidase in the presence of atmospheric oxygen and cytochrome c oxidized phenyl diamine to indofenol in the form of violet coloration. After the completed tests for Acinetobacter spp., no discoloration was observed. Further identification was carried out in an automatic VITEK 2 Compact analyzer (BioMerieux, France).

Determination of antimicrobial susceptibility of isolated microorganisms was carried out by means of three methods: disc diffusion antibiotic sensitivity testing (Kirby-Bauer), in which discs were used (in Poland - Oxoid - UK, Ukraine - HiMedia - India). In Poland, two methods were used: a gradient-diffusion E-test and automatic Vitek 2 Compact, where cards for antimicrobial susceptibility testing for Gramnegative strains (bioMerieux) were applied.

Also in Poland, isolates of Enterobacteriaceae, Pseudomonas aeruginosa and Acinetobacter baumannii insensitive to carbapenems were tested with EDTA (Ethylene-DiamineTetra-Acetate) towards KPC (Klebsiella pneumoniae carbapenemase) and test OXA-48 with temocilin.

The method of determining drug susceptibility as well as other resistance mechanisms and their interpretation were performed in accordance with EUCAST recommendations and the manufacturer's test methodology.

\section{Recommended antimicrobial therapy schemas for pneumonia associated with mechanical ventilation}

Recommended empirical treatment used in the analyzed countries. According to the published "Recommendations for the diagnosis, treatment and prevention of antibioticassociated infections in hospitals" or "Guidelines for the Management of Adults with Hospital-acquired, VentilatorAssociated and Healthcare-Associated Pneumonia", the etiological factor of infection and risk factors should be considered, i.e. general condition of the patient, renal failure, liver and other health disorders, which are of great importance in the choice and dosage of antibiotics $[12,13,14,15]$. In each case, the risk of infection with a multi-resistant microorganism, which increases with the patient's hospitalization time, prior antibiotic therapy, and whether the patient has undergone invasive treatment or is in Intensive Care, should be considered.

In the case of no efficacy of the empiric therapy, the diagnosis, the factors that burden the hospitalized and the results of the microbiological examination, should be re-examined. The choice of empirical treatment depends on the differentiation of early and late VAP. In patients with early VAP (early VAP), the most common etiologic factors that proved to occur during the first four days of respiratory distress are S. aureus 
(more often, methicillin-sensitive Staphylococcus aureus), $S$. pneumoniae, $H$. influenzae and relatively antibiotic-resistant Gram-negative strains. The proposal for empirical treatment in early VAP in a patient without risk factors for infection with multidrug resistant organisms is: ceftriaxone $1 \times 2 \mathrm{~g}$ iv or levofloxacin $1 \times 750 \mathrm{mg}$ iv. [12].

The most frequently infectious microorganisms and those causing late VAP (late-onset VAP) that develop during more than four days of respirator use are the most commonly encountered multidrug resistant hospital lines: Klebsiella pneumoniae, Escherichia coli and other Enterobacteriaceae strains, Pseudomonas aeruginosa, Acinetobacter baumannii, Stenotrophomonas maltophilia and Staphylococcus aureus (MRSA - methicyllin-resistant Staphylococcus aureus). Anaerobic bacteria are a rare cause of VAP. In ICU, lung inflammation may be caused by more than one species of microorganisms.

Selection of empirical antibiotic VAP should be based on local epidemiological data. The choice of empirical antibiotic therapy in late VAP includes: ceftazidime: $3 \times 2 \mathrm{~g}$ iv or piperacillin with tazobactam $4 \times 4.5 \mathrm{~g}$ iv. In very severe conditions: imipenem $4 \times 0.5 \mathrm{~g}$ iv or $3-4 \times 1 \mathrm{~g}$ iv or meropenem $3 \times 1 \mathrm{~g}$ iv. If the microscopic slide from a sample of the lower respiratory tract is mainly Gram-positive, glycopeptide or linezolid should be added. Treatment time: 8 days, longer treatment is recommended if infection is caused by $P$. aeruginosa or S. aureus [12].

\section{RESULTS}

BAL studies under microbiological conditions. Comparative evaluation of the isolation frequency of ESKAPE bacterial strains in materials obtained from patients in Poland and Ukraine (Figure 1).

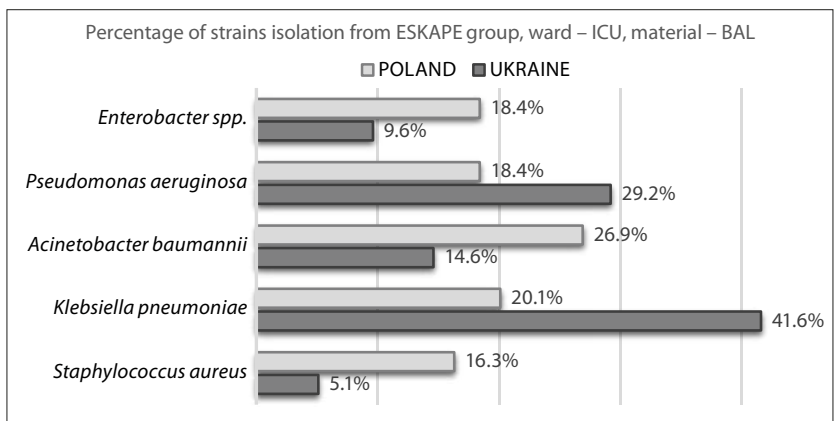

Figure 1. Comparative evaluation of the isolation frequency of ESKAPE bacterial strains in materials obtained from patients in Poland and Ukraine

From BAL, mainly Gram-negative strains were isolated. Among the patients in Poland, A. baumannii strains (26.9\%) were dominant. The percentage of patients from Ukraine with species A. baumannii was $14.6 \%$. Patients from Ukraine were prevalent in K. pneumoniae (41.6\%), followed by P. aeruginosa (29.2\%). K. pneumoniae was the species with the highest prevalence compared to other bacteria. Participation in $S$. aureus infections on both the Polish and Ukrainian sides was $16.3 \%$ vs. $5.1 \%$. In the current study, the species Enterococcus spp. was omitted, because current recommendations have failed to confirm that this microorganism can cause VAP $[12,13,14,15]$.

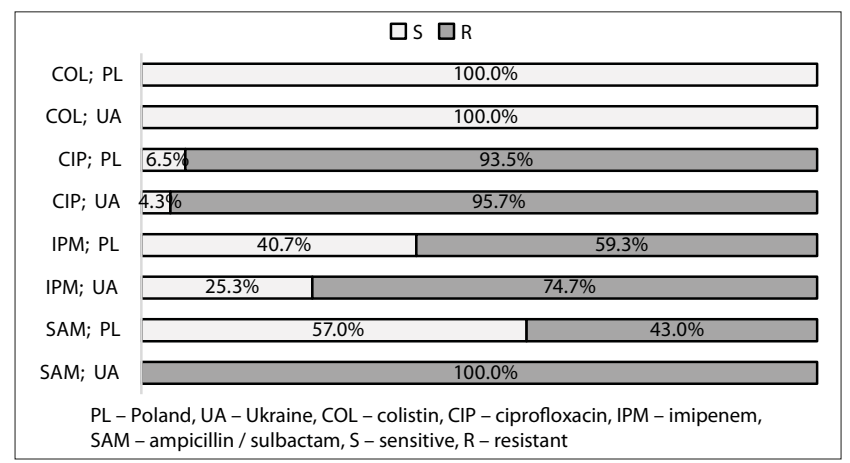

Figure 2. Evaluation of drug resistance / susceptibility profile of A. baumannii strains for selected drugs

Drug resistance / susceptibility to A. baumannii strains. The investigated isolates of $A$. baumannii, the degree of drug resistance to selected drugs was set.

During the considered period, the analyzed A. baumannii strains in Ukraine proved to be significantly more resistant to drugs, compared to isolates from Poland. Undisputed results concerning the differences in antibiotic resistance in Poland and in Ukraine were mainly due to the total resistance of Acinetobacter isolates to ampicillin with sulbactam found in $100 \%$ isolates in Ukraine vs. $43 \%$ in Poland. Since sulbactam, as the only $\beta$-lactamase inhibitor has activity against strains of $A$. baumannii species $[16,17]$, the activity was determined in this study against this microorganism. Afterwards, a significant problem was the high resistance to imipenem, including the much higher resistance of strains in Ukraine, which led to the analysis of the impact of antibiotic policy on this condition in Poland and Ukraine.

It is interesting that in the case of resistance to a representative of fluoroquinolones - ciprofloxacin, a similar level of resistance was shown: 95.7\% (UA) vs. 93.5\% (PL).

All strains of A. baumannii isolated in Poland and Ukraine were sensitive to colistin.

Risk factors of microbial infections from ESKAPE group. According to literature data, the risk factors of infections caused by ESKAPE pathogens [18] include the following: age above 65 years of age, hospitalization in the previous year and previous antibiotic treatment. Data on the incidence of infection risk factors in each facility are presented in Table 1.

Table 1. Risk factors of infection caused by multidrug-resistant microorganisms

\begin{tabular}{lcc}
\hline RISK FACTOR & POLAND & UKRAINE \\
\hline average number of hospitalization days in a ward & 25 days & 19 days \\
\hline average age & 63 years & 49 years \\
\hline extreme respiratory failure & $81 \%$ & $88 \%$ \\
\hline circulation failure & $62 \%$ & $68 \%$ \\
\hline renal failure & $15 \%$ & $32 \%$ \\
\hline diabetes & $18 \%$ & $14 \%$ \\
\hline obesity & $6 \%$ & $6 \%$ \\
\hline active cancer disease & $7 \%$ & $4 \%$ \\
\hline $\begin{array}{l}\text { alcoholism } \\
\text { nicotinism }\end{array}$ & $8 \%$ & $10 \%$ \\
\hline $\begin{array}{l}\text { received antibiotic therapy up to } 3 \text { month before } \\
\text { admission to hospital }\end{array}$ & $60 \%$ & $82 \%$ \\
\hline $\begin{array}{l}\text { patients with a severe general condition directly transferred } \\
\text { from another hospital or ward during the preceding period } \\
\text { of VAP infection }\end{array}$ & $74 \%$ & $78 \%$ \\
\hline
\end{tabular}


In the analyzed Intensive Care Units, the average number of hospitalization days in a ward in Poland was 25 days, while in Ukraine it was 19 days. A difference was also observed in the average age of patients (PL) 63 years vs. (UA) 49 years.

There was a relationship between the duration of hospitalization combined with the prior use of broad spectrum antibiotic treatment, as well as the acquisition and selection and the occurrence of infections caused by drug resistant strains, as confirmed by other authors $[2,6,8$, 10]. Self-observation also confirms that antibiotic treatment received up to three months before admission to hospital is very likely to be associated with infections caused by multidrug resistant microorganisms (60\% vs. $82 \%)$.

The highest percentage of patients, both in Poland and Ukraine, was associated with a risk factor for severe respiratory distress ( $81 \%$ vs. $88 \%)$, circulation failure $(62 \%$ vs. $68 \%$ ), and admission of patients to ICU in a very severe condition from other wards or hospitals ( $74 \%$ vs. $78 \%$ ).

Evaluation of prospects for the development of infections caused by highly resistant bacteria. One of the major dangers to public health worldwide is the increase in microbial resistance to antimicrobial drugs. In addition, for several years there has been an increase in infections caused by microorganisms not related to ESKAPE, mainly Clostridium difficile and drug resistant bacteria from the Enterobacteriaceae family (other than Klebsiella pneumoniae and Enterobacter spp.); hence, changing the acronym ESCAPE has been proposed [19].

Another epidemiological threat is infiltration of these stains to the outside environment and the possibility of causing infections.

Data from the literature suggests that, in addition to ESKAPE pathogens, the species Stenotrophomonas maltophilia should be included as a microorganism that causes more and more frequent infections in healthcare settings $[1,20,21,22]$.

\section{DISCUSSION}

Due to the rapidly growing threat of multi-drug resistant microorganisms, a continuous surveillance of the epidemiological situation in health care facilities is essential. An indispensable tool for observation and response in this area is the rapid diagnosis of infections, which is also crucial in the choice of empirical therapy. One must not ignore the ongoing medical education of spreading and preventing infections. These activities should be covered by the appropriate antibiotic policy programme, considering the modification of these recommendations to local use.

An important aspect is the dosage and the time of administration of antibiotics that should be adapted to the course of the disease to minimize the danger of the selection and spread of resistant strains, especially in the hospital environment.

The presented study showns, among other things, that the most common cause of A. baumannii infection are risk factors, such as: previous antibiotic therapy, severe general condition of the patient, extreme respiratory failure, circulatory failure and previous hospitalization. In these patients, these factors have caused pharmacokinetic disorders and, as a result, decreased effectiveness of antibiotic therapy. Changes in absorption, distribution or elimination in the patient's body preclude proper dosing of the drugs. Consequently, modification of the dosage of antibiotics most often results in a too low drugs concentration, below the required minimal inhibitory concentration (MIC-minimal inhibitory concentration) [11].

The results from the study suggest that the problem with ESKAPE pathogens, including Acinetobacter baumannii, in both hospitals is associated with multidrug-resistant bacteria. This is probably due to the specificity of the operation of anaesthesia and intensive care units, and the increased pressure exerted by the large amount of antimicrobial drugs.

\section{CONCLUSIONS}

- The divergence in the phenotypes of A. baumannii drug resistance in the studied countries may be different due to the diversified antibiotic policies applied in the country concerned.

- Thehigh resistancelevel of $A$. baumannii to fluoroquinolones which represented itself on a similar level (>90\%) both in Poland and Ukraine, as well as resistance to ampicillin with sulbactam of all A. baumannii cultures from Ukraine, are most likely due to the unreasonable use of these drugs in hospital and outpatient treatment.

- All A. baumannii isolates from Poland and Ukraine showed sensitivity to colistin, which was the last treatment option in severe infections.

- The scheme of the recommended empirical treatment in the discussed countries was very similar, mainly based on developed recommendations in foreign literature.

- The occurrence of individual risk factors for infection with multidrug-resistant microorganisms was found to be at a similar level in the analyzed countries.

\section{REFERENCES}

1. Renner LD, Zan J, Hu LI, Martinez M, Resto PJ, Siegel AC, Torres C, Hall SB, Slezak TR, Nguyen TH, Weibel DB. Detection of ESKAPE Bacterial Pathogens at the Point of Care Using Isothermal DNA-Based Assays in a Portable Degas-Actuated Microfluidic Diagnostic Assay Platform. Appl Environ Microbiol. 2017; 83(4).

2.Hubick S, Jayaraman A, McKeen A, Reid S, Alcorn J, Stavrinides J, Sterenberg BT. A potent synthetic inorganic antibiotic with activity against drug-resistant pathogens. Sci Rep. 2017; 7: 41999.

3. Meeker DG, Jenkins SV, Miller EK, Beenken KE, Loughran AJ, Powless, Muldoon TJ, Galanzha EI, Zharov VP, Smeltzer MS, Chen J. Synergistic Photothermal and Antibiotic Killing of Biofilm-Associated Staphylococcus aureus Using Targeted Antibiotic-Loaded Gold Nanoconstructs. ACS Infect Dis. 2016; 2(4): 241-250.

4. Bales PM, Renke EM, May SL, Shen Y, Nelson DC. Purification and Characterization of Biofilm-Associated EPS Exopolysaccharides from ESKAPE Organisms and Other Pathogens. PLoS One. 2013; 8(6).

5. Behroozian S, Svensson SL, Davies J. Kisameet Clay Exhibits Potent Antibacterial Activity against the ESKAPE Pathogens. MBio. 2016; 7(1). 6. Bonnin RA, Poirel L, Nordmann P. New Delhi metallo- $\beta$-lactamaseproducing Acinetobacter baumannii: a novel paradigm for spreading antibiotic resistance genes. Future Microbiol. 2014; 9(1): 33-41.

7.Lob SH, Hoban DJ, Sahm DF, Badal RE. Regional differences and trends in antimicrobial susceptibility of Acinetobacter baumannii. Int J Antimicrob Agents. 2016; 47(4): 317-23.

8. Santajit S, Indrawattana N. Mechanisms of Antimicrobial Resistance in ESKAPE Pathogens. Biomed Res Int. 2016; 2016: 2475067.

9. Bionda N, Fleeman RM, de la Fuente-Nunez C, Rodriguez MC, Reffuveille F, Shaw L, Pastar I, Davis SC, Hancock RE, Cudic P. Identification of novel cyclic lipopeptides from a positional scanning 
combinatorial library with enhanced antibacterial and antibiofilm activities. Eur J Med Chem. 2016; 108: 354-63.

10. Hsu LY, Apisarnthanarak A, Khan E, Suwantarat N, Ghafur A, Tambyah PA. Carbapenem-Resistant Acinetobacter baumannii and Enterobacteriaceae in South and Southeast Asia. Clin Microbiol Rev. 2017; 30(1): 1-22.

11. Bassetti M, Luyt CE, Nicolau DP, Pugin J. Characteristics of an ideal nebulized antibiotic for the treatment of pneumonia in the intubated patient. Ann Intensive Care. 2016; 6(1): 35.

12. Hryniewicz W, Ozorowski T. Recommendations for the diagnosis, antibiotic treatment and prevention of infections in hospitals. 2015. http://antybiotyki.edu.pl (in Polish).

13. Masterton RG, Galloway A, French G, et al. Guidelines for the management of hospital-acquired pneumonia in the UK: Report of the Working Party on Hospital-Acquired Pneumonia of the British Society for Antimicrobial Chemotherapy. J Antimicrob Chemother. 2008; 62: 5-34.

14. Rotstein C, Evans G, Born A, et al. Clinical practice guidelines for hospital-acquired pneumonia and ventilator-associated pneumonia in adults. Can J Infec Dis Med Microbiol. 2008; 19: 19-53.

15. American Thoracic Society, Infectious Diseases Society of America Guidelines for the management of adults with hospital-acquired, ventilator-associated, and healthcare-associated pneumonia. Am J Respir Crit Care Med. 2005; 171: 388-416.
16. Penwell WF, Shapiro AB, Giacobbe RA, Gu RF, Gao N, Thresher J, McLaughlin RE, Huband MD, DeJonge BL, Ehmann DE, Miller AA. Molecular mechanisms of sulbactam antibacterial activity and resistance determinants in Acinetobacter baumannii. Antimicrob Agents Chemother. 2015; 59(3): 1680-9.

17. Fishbain J, Peleg AY. Treatment of Acinetobacter Infections. Clin Infect Dis (2010) 51(1): 79-84.

18. Cardoso T, Ribeiro O, Aragao IC, Costa-Pereira A, Sarmento AE. Additional risk factors for infection by multidrug-resistant pathogens in healthcare-associated infection: a large cohort study. BMC Infect Dis. 2012; 12: 375.

19. De Rosa FG, Corcione S, Pagani N, Di Perri G. From ESKAPE to ESCAPE, from KPC to CCC. Clin Infect Dis. 2015; 60(8): 1289-90.

20. Mogyorodi B, Dunai E, Gal J, Ivanyi Z. Ventilator-associated pneumonia and the importance of education of ICU nurses on prevention Preliminary results. Interv Med Appl Sci. 2016; 8(4): 147-151.

21. Chung H, Lieberman TD, Vargas SO, Flett KB, McAdam A, Priebe GP, Kishony R. Global and local selection acting on the pathogen Stenotrophomonas maltophilia in the human lung. Nat Commun. 2017; 8: 14078

22. Ebara H, Hagiya H, Haruki Y, Kondo E, Otsuka F. Clinical Characteristics of Stenotrophomonas maltophilia Bacteremia: A Regional Report and a Review of a Japanese Case Series. Intern Med. 2017; 56(2): 137-142. 